\section{Latinoamericanos en proyectos europeos: asimetrías en la cooperación científica}

Resumen: En este artículo se discute la cooperación científica internacional en un sentido muy específico: las actividades de cooperación entre contextos de mayor desarrollo y espacios semi-periféricos. Para ello se analiza la participación de grupos de investigación latinoamericanos de Brasil, Argentina, México, Chile y Colombia en los consorcios financiados por el 7 mo Programa Marco de la Unión Europea. A través de este análisis se indaga acerca de las razones por las cuales la participación de grupos latinoamericanos es cada vez más activa, y sobre todo, qué hacen estos grupos cuando participan de consorcios internacionales, cómo se organizan y se distribuye el trabajo, y cuáles son los grados de libertad de los científicos latinoamericanos en un mundo cada vez más globalizado.

Palabras clave: Cooperación científica internacional, Centros y Periferias, Europa y América Latina.

Latin Americans in European projects: asymmetries in international scientific cooperation

Abstract: This article discusses the international scientific cooperation in a very specific sense: the cooperative activities between more developed contexts and semi-peripheral spaces. Therefore, the participation of Latin American research groups from Brazil, Argentina, Mexico, Chile and Colombia in the consortia financed by the 7th Framework Program of the European Union is analyzed. Through this analysis, we ask about the reasons for the increasingly active participation of Latin American groups, especially, the kind of work these groups carry out when they participate in international consortia, how the work is organized and distributed, and what the degrees of freedom for Latin American scientists are in an increasingly globalized world.

Key words: International Scientific cooperation, Centers and Peripheries, Europe \& Latin America.

Latino-americanos em projetos europeus: assimetrias na cooperação científica internacional

Resumo: Este artigo discute a cooperação científica internacional em um sentido muito específico: atividades de cooperação entre contextos de maior desenvolvimento e espaços semi-periféricos. Para isso, é analisada a participação de grupos de pesquisa latino-americanos do Brasil, Argentina, México, Chile e Colômbia nos consórcios financiados pelo $7^{\circ}$ Programa-Quadro da União Européia. Por meio dessa análise, são investigadas as razões pelas quais a participação de grupos latino-americanos é cada vez mais ativa e, sobretudo, o que esses grupos fazem quando participam de consórcios internacionais, como o trabalho é organizado e distribuído e quais são os graus de liberdade dos cientistas latino-americanos em um mundo cada vez mais globalizado. Palavras-chave: Cooperação científica internacional, centros e periferias, Europa e América Latina.

\section{Ciencia Tecnología y Política \\ Año 3 N$^{\circ} 4$ Mayo 2020}

Adriana Feld
Historiadora. Dra. en
Ciencias Sociales.
Centro CTS. Universidad
Maimónides, CONICET y
Universidad Nacional de
San Martín
feldri75@yahoo.com.mx
Pablo Kreimer
Sociólogo. Dr. en "Science,
technologie et société”.
Centro CTS. Universidad
Maimónides, CONICET
y Universidad Nacional
de Quilmes
pkreimer@yahoo.com

Fecha de recibido: $12 / 03 / 2020$

Fecha de aprobado: 23/04/2020

https://doi.org/10.24215/26183188e035

https://revistas.unlp.edu.ar/CTyP

ISSN 2618-3188

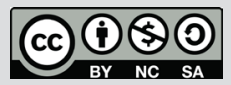

Esta obra está bajo licencia Creative Commons Atribución-NoComercial-Compartirlgual 4.0 Internacional http://creativecommons.org/licenses/bync-sa/4.O/deed.es_AR 


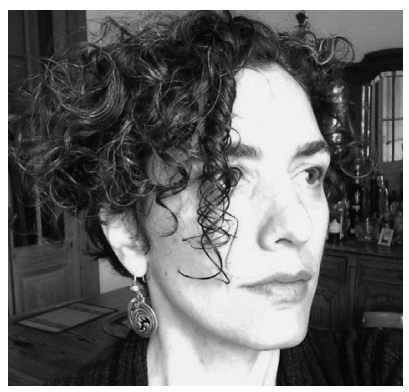

Adriana Feld

Historiadora.

Dra. en Ciencias Sociales.

Centro CTS. Universidad Maimónides, CONICET y Universidad Nacional de San Martín feldri75@yahoo.com.mx

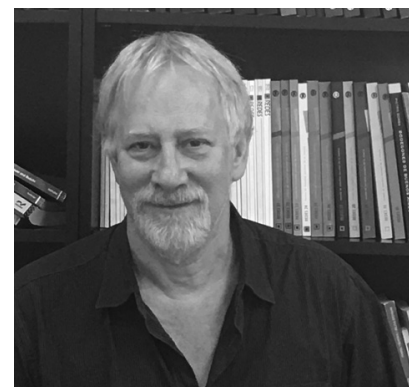

Pablo Kreimer

Sociólogo. Dr. en "Science, technologie et société". Centro CTS. Universidad Maimónides, CONICET y Universidad Nacional de Quilmes

pkreimer@yahoo.com

\section{Latinoamericanos en proyectos europeos: asimetrías en la cooperación científica internacional}

Resumen: En este artículo se discute la cooperación científica internacional en un sentido muy específico: las actividades de cooperación entre contextos de mayor desarrollo y espacios semi-periféricos. Para ello se analiza la participación de grupos de investigación latinoamericanos de Brasil, Argentina, México, Chile y Colombia en los consorcios financiados por el $7 \mathrm{mo}$ Programa Marco de la Unión Europea. A través de este análisis se indaga acerca de las razones por las cuales la participación de grupos latinoamericanos es cada vez más activa, y sobre todo, qué hacen estos grupos cuando participan de consorcios internacionales, cómo se organizan y se distribuye el trabajo, y cuáles son los grados de libertad de los científicos latinoamericanos en un mundo cada vez más globalizado.

\section{Introducción}

Durante muchas décadas, la cooperación internacional fue considerada como "algo bueno en sí mismo", como un indicador de modernidad de la ciencia local, o de cosmopolitismo de sus investigadores. En buena medida, esa concepción sigue inspirando a buena parte de las políticas científicas de los países latinoamericanos, que suelen tener una visión acrítica de la cooperación internacional, bajo la fórmula de "cuantos más vínculos internacionales tengan nuestros investigadores, mejor".

En términos analíticos, sin embargo, la cuestión ha sido problematizada desde diferentes perspectivas. Así, al tiempo que la intensidad de las cooperaciones científicas fue aumentando en forma impresionante en los últimos tiempos, algunos autores comenzaron a cuestionar que el impacto de las colaboraciones tenga sólo un aspecto positivo 
sobre el desarrollo de los campos científicos locales (Vessuri, 1996, Kreimer, 2019). Sobre todo, se ha puesto el acento en el papel de las asimetrías, particularmente en la medida en que participen científicos de contextos calificados como "centrales" por su desarrollo económico y científico (no cabe duda de que ambos desarrollos evidencian una alta correlación).

Por ello, en este artículo analizamos un tipo particular de cooperación científica internacional: aquella emprendida por individuos o grupos latinoamericanos que participaron en proyectos financiados por la Unión Europea a través del instrumento de los Programas Marco, y con el aporte de fondos de contraparte de los gobiernos de países latinoamericanos a los que pertenecen los investigadores.

Visto en perspectiva histórica, hemos podido constatar que uno de los componentes de las políticas científicas de la mayor parte de los países en desarrollo ha consistido en estimular la participación de científicos locales en emprendimientos conjuntos con sus pares de los países más avanzados. Estas colaboraciones tienen muchas manifestaciones: a comienzos del siglo pasado (e incluso antes), lo más frecuente era invitar a científicos europeos o estadounidenses a radicarse en forma temporal o permanente en contextos periféricos como un modo de impulsar nuevos campos, o de modernizar los existentes. Más adelante se generalizó el envío de jóvenes científicos a formarse en centros prestigiosos del extranjero o el diseño de proyectos conjuntos, entre otros instrumentos (Katz y Martin, 1997). Más recientemente, desde fines del siglo pasado, se fueron generando grandes redes internacionales que funcionan como consorcios de investigación, y de las que suelen participar desde unas decenas hasta miles de investigadores. Estas redes, que en textos precedentes hemos denominado "Mega-ciencia" (Kreimer, 2019), son financiadas por instituciones y agencias de los países más desarrollados en función de las prioridades estratégicas establecidas por los gobiernos de dichos países, o por instancias supranacionales (como la Unión Europea, la Organización Mundial de la Salud, la Organización de las Naciones Unidas para la Alimentación y la Agricultura, etc.).

Tal como ya ha sido suficientemente documentado, la cooperación entre científicos de diversos países, medida a través de las publicaciones, no ha cesado de aumentar, en particular en las últimas 3 décadas (Adams, 2012 y 2013). Leydesdorf y Wagner (2008) señalan que "si se miden las coautorías, hay un crecimiento lineal entre 1990 y 2005 en términos del número de papers" (pp. 317-18), pero que creció en forma "exponencial en términos del número de domicilios internacionales", lo que confirmaría la hipótesis de Persson et al. (2004) sobre una inflación de las colaboraciones internacionales. La proporción de artículos escritos en co-autoría internacional versus los artículos "domésticos" (aquéllos firmados por investigadores de un mismo país) es cada vez mayor en algunos países centrales (por ejemplo, en el Reino Unido o en Suiza, es ya más de la mitad), mientras que en algunos países de menor producción científica ya es imposible distinguir la "ciencia nacional" (Adams, 2013). En 1988, poco más de un $10 \%$ de los papers estaban firmados por investigadores de más de un país. Veinte años más tarde, ese porcentaje había ascendido al 30\% (Boekholt, Edler, Cunningham y Flanagan, 2009; Gaillard, Gaillard y Arvanitis, 2010).

En ese contexto, no llama la atención que la cooperación de científicos latinoamericanos en proyectos desarrollados junto con grupos y colegas de países más desarrollados haya aumentado en forma muy significativa durante los últimos años (Gaillard y Arvanitis, 2013; Kreimer y Levin, 2013).

En este artículo hacemos un abordaje diferente de 
aquellos que analizaron la cooperación científica internacional a través de métodos bibliométricos, ya que ello presenta muchas limitaciones para explicar los aspectos que nos parecen más significativos. Por ejemplo, quienes analizaron las colaboraciones científicas de América Latina, como Cardoza y Fornés (2011), De Filippo, Morillo y Fernández (2008), Gómez, Fernández y Sebastián (1999), Miguel y Moya-Anegón (2009), están naturalmente restringidos a recoger indicadores tales como coautorías, que son indicadores indirectos de la cooperación, ya que ésta es un fenómeno multidimensional: aparecer como coautor en un artículo científico puede reflejar muchas circunstancias diferentes, desde una colaboración bien estructurada hasta una relación esporádica o incidental, pasando por ser el proveedor de material necesario para la investigación o desarrollar algún método en particular.

Aquí consideramos 4 aspectos específicos:

- Nos concentramos en la cooperación científica que generalmente se ha denominado "Norte-Sur", o entre países centrales y contextos semi-periféricos o "no hegemónicos" (Losego y Arvanitis, 2008, Kreimer, 2006). ${ }^{1}$

- Observamos, dentro de este tipo de relaciones, a la cooperación entre América Latina y Europa, puesto que se dispone de información contenida en bases de datos completas y bien estructuradas. ${ }^{2}$

- Consideramos la cooperación en el marco de proyectos institucionalizados cuyo financiamiento lo provee la Unión Europea y en donde participan gru- pos latinoamericanos. Ello implica hacer un recorte metodológico explícito, puesto que somos conscientes de que no todas las relaciones de cooperación internacional se dan bajo un paraguas de programas de cooperación formal.

- A diferencia de la mayor parte de los estudios sobre cooperación internacional, no trabajaremos sobre publicaciones (co-autorías u otras) sino que proponemos un estudio cualitativo sobre las percepciones y motivaciones de los actores involucrados.

El objetivo del texto es poner en cuestión e indagar empíricamente las afirmaciones corrientes que consideran a la cooperación científica como algo "bueno en sí mismo" y como una contribución a un proceso según el cual "en general, se acepta que los países en desarrollo deben fortalecer sus capacidades para generar y explotar tecnología que resuelva sus problemas de producción, para alimentar a su población, o para cuidar la salud y educación de sus pueblos, y hacerlo de manera sostenible." (Velho, 2002: 26).

Pretendemos encuadrar nuestro estudio sobre las cooperaciones científicas en el análisis de las relaciones entre "centros" y "periferias" (enfatizando ambos plurales, Kreimer, 2006 y 2019) dentro de un mundo cambiante y globalizado.

En nuestro análisis consideramos la participación de grupos de investigación de los cinco países más dinámicos de América Latina (Argentina, Brasil, Chile, Colombia y México) en proyectos de ciencias exactas, naturales y tecnológicas del sub-programa

\footnotetext{
1 La definición de cooperación "Norte-Sur" es engañosa, puesto que traslada en forma mecánica una definición geográfica a las capacidades de cada contexto regional. En sentido estricto, México está en el Norte y Australia en el Sur. Mencionamos la expresión, porque sigue estando fuertemente anclada en los discursos, pero la definición "periférico" o "no hegemónico" nos parece mucho más adecuada a nuestro objeto.

2 Naturalmente, hay otras formas de cooperación científica entre europeos y latinoamericanos que no están incluidas en estos programas: por un lado, la cooperación organizada gracias a programas bilaterales y, por otro, consorcios financiados por organismos internacionales u otro tipo de agencias. Finalmente, hay una proporción importante de cooperación informal, que no queda necesariamente registrada en documentos oficiales.
} 
"cooperación" de la Unión Europea, que concentra dos tercios del presupuesto del $7^{\circ}$ Programa Marco. ${ }^{3}$ La elección de estos cinco países se debe a que, en su conjunto, representan más del $90 \%$ de la participación latinoamericana en consorcios europeos.

La pregunta general se dirige, sobre todo, a comprender por qué los consorcios de investigación europeos incorporan grupos de investigación de América Latina. Ello se declina, a su vez, en otras preguntas: a) en el plano de las estrategias cognitivas, ¿cuáles son las motivaciones de los líderes de proyectos europeos para incluir grupos de América Latina en la conformación de los consorcios de investigación?; b) en el plano de las prácticas, ¿cuál es el rol de los grupos latinoamericanos en dichos consorcios? A ello subyace otra cuestión, que sólo abordamos parcialmente en este texto: c) en el plano político-institucional, ¿cuál es el interés de la Unión Europea en fomentar la cooperación con América Latina?

El propósito es que, a través de esta lectura crítica, podamos aportar elementos para repensar las políticas de cooperación científica internacional de los países latinoamericanos, de modo que la participación en grandes consorcios pueda ser reorientada hacia las agendas localmente relevantes. Luego de una breve nota metodológica, presentamos los principales resultados obtenidos, a lo que le sigue el análisis y las conclusiones.

\section{Nota Metodológica ${ }^{4}$}

Nuestro trabajo empírico se basa en el análisis de proyectos del sub-programa cooperación del $7^{\circ}$ Programa Marco (FP7), financiado por la Unión
Europea. En una primera instancia hemos extraído, del total de las bases de datos, aquellos proyectos en los que se registró la participación de, por lo menos, un grupo de Argentina, Brasil, México, Chile o Colombia. En segunda instancia, hemos organizado esta muestra por área temática y seleccionado aquellas áreas en las que América Latina tiene mayor tradición de investigación: salud, y bioeconomía basada en el conocimiento (KBBE, por sus siglas en inglés). A ellas agregamos otra que, a diferencia de las anteriores, implica un tipo de investigación sobre el terreno (por contraposición a la investigación de laboratorio) destinada en general al estudio de sistemas globales (environment-ENV-). Posteriormente, se seleccionaron entre cinco y seis proyectos de cada una de las tres áreas, que cumplieran con los siguientes requisitos: que estuvieran financiados con el esquema "proyectos colaborativos" ${ }^{\text {y }}$ y que abordaran tópicos diferentes dentro del área, de modo de tener representados proyectos con diversas orientaciones.

Luego, identificamos a los coordinadores de los proyectos seleccionados (17 en total), a quienes se les realizó una entrevista semi-estructurada, en la que se apuntó a indagar las cuestiones referidas al origen del proyecto, motivaciones para convocar a los grupos latinoamericanos, distribución de las actividades, asignación de responsabilidades, resultados obtenidos por el consorcio, rol de las empresas participantes, y beneficios para los grupos de América Latina.

En el análisis hacemos referencia a los proyectos con la clave del área (Salud, KBBE, ENV) y al número de proyectos según nuestra muestra, del 1 al 6.

\footnotetext{
${ }^{3}$ Los Programas Marco son los instrumentos más importantes que utilizó la Unión Europea para financiar la investigación entre los años 1984 y 2013.

${ }^{4}$ Por razones de espacio, no podemos desarrollar in extenso la metodología utilizada y su justificación, por lo que presentamos en esta sección un breve resumen de la misma.

${ }^{5}$ Nos concentramos en proyectos estrictamente de investigación, descartando el financiamiento para la conformación y/o consolidación de redes internacionales, donde la participación latinoamericana es menos relevante.
} 


\section{Resultados y análisis}

Para concentrar el análisis, organizamos los resultados en dos categorías principales: motivaciones de los líderes europeos para enrolar a los grupos latinoamericanos, y actividades desarrolladas por estos últimos, dentro de la división interna del trabajo.

Motivaciones para enrolar grupos latinoamericanos

Lo primero que observamos en las respuestas de los líderes europeos en relación con las motivaciones para incluir a grupos latinoamericanos en los consorcios es que, por regla general, existe una alta valoración de la calidad científica de los científicos de América Latina convocados. Ello no debe sorprendernos porque, tal como hemos mostrado en trabajos anteriores, los grupos de élite latinoamericanos muestran una alta correlación en su grado de prestigio relativo y en su grado de internacionalización (Kreimer, 2006). Dicho de otro modo, los líderes científicos latinoamericanos construyen su prestigio en parte en su desempeño local, pero sobre todo en virtud de sus relaciones internacionales, que les otorgan capital simbólico en el plano local.

Incluso, en el pasado, cuando aún la publicación de artículos científicos en revistas internacionales con referato no se había institucionalizado como una medición estándar de la calidad científica, parte del prestigio local se ganaba a partir del reconocimiento de pares internacionales. En la actualidad, participar en proyectos internacionales -o financiados con fondos internacionales- es una fuente de prestigio importante en todos los países, pero ello es más evidente en los de desarrollo científico intermedio.

Hay, sin embargo, diferencias sustantivas en cuanto a los estímulos para enrolar grupos latinoamericanos. Hemos identificado 4 tipos de motivaciones:

a) La inclusión de latinoamericanos como condición para la obtención del subsidio.

Es el caso de Salud-5; KBBE-6 y ENV-6. En estos casos cabe preguntarse por la motivación en la elección de esos grupos en vez de otros, pero no por qué incluir a un grupo latinoamericano. La respuesta aquí parece venir de dos fuentes diferentes: la primera, de tipo técnico-cognitiva, como las capacidades adicionales que pueden proveer los grupos latinoamericanos invitados, o el acceso a recursos cognitivos, como la disponibilidad de cepas específicas, el acceso a enfermos, etc. La segunda es de orden sociológico, y refiere a los vínculos establecidos entre los partenaires en el pasado, los lazos de familiaridad y de confianza, e incluso, los paradigmas científicos compartidos, que son frecuentemente evocados por los entrevistados.

b) Consorcios que trabajan sobre cuestiones latinoamericanas

Es el caso de la mayor parte de los proyectos de salud, que incluyen enfermedades tropicales (como la enfermedad de Chagas): SALUD-1, 4 y 5 . En este caso la pregunta adicional que debemos formular es: "¿por qué Europa decide financiar la investigación sobre temas que no afectan (o afectan sólo marginalmente) su propio contexto?". Aquí también encontramos dos niveles de respuesta diferente: el primero refiere a lo que la investigación sobre cuestiones locales (en este caso, enfermedades, pero naturalmente hay otros tópicos) puede aportar a la comprensión de fenómenos de alcance más fundamental. En este sentido, por ejemplo, la investigación sobre búsqueda de blancos para atacar a los agentes causales de esas enfermedades puede dar pistas sobre mecanismos biológicos o fisiológicos fundamentales, que serían extrapolables a otros aspectos cognitivos por fuera de los casos estudiados. Además, la prueba con moléculas novedosas puede generar, en asociación con empresas del sector farmacéutico, conocimientos aplicables a enfermedades de tipo universal, autonomizándose de las cuestiones exclusivamente locales. 
El segundo nivel de respuesta refiere al resultado de los procesos de globalización y de las corrientes migratorias: algunas enfermedades clásicamente "tropicales" se fueron extendiendo hacia otras regiones y generan nuevos problemas frente a los cuales hay muy poco conocimiento acumulado. Este es el caso, por ejemplo, de la enfermedad de Chagas en España, Francia o los estados de Texas y California en los Estados Unidos. En estos casos, además del prestigio que los investigadores latinoamericanos puedan detentar, se suma una expertise específica en relación con un objeto de estudio, y el acceso exclusivo a recursos indispensables para la investigación, como los pacientes, o las diversas cepas de parásitos u otros organismos.

c) Consorcios que investigan problemas globales, pero que tienen manifestaciones específicas en diversos contextos

Aquí se necesitan diversos puntos de observación localizados en contextos diferentes. Este es el caso más frecuente en los proyectos relacionados con el medio ambiente y, en nuestro estudio, en los consorcios ENV-1, 2 y 4 . En estos casos, las situaciones que se investigan responden a fenómenos de orden más general, como el cambio climático, y tienen manifestaciones en algunos sitios geográficos bien localizados, como la concentración de mercurio en los ríos, o las consecuencias del derretimiento de los glaciares. En estos casos, la incorporación de grupos latinoamericanos responde a dos modalidades complementarias: la primera es la necesidad de recolectar datos en puntos de observación específicos que generen una "mirada global" del problema. Por lo tanto, los grupos latinoamericanos deben tener un acceso a esos recursos -ríos, glaciares, diversas especies de fauna o flora, etc. - y contar con la capacidad técnica para generar datos en forma estandarizada según los protocolos establecidos por el conjunto del consorcio, ya que aquí el uso de la metodología es clave para la homogeneidad de los datos recogidos.

La segunda modalidad se refiere a que los grupos latinoamericanos deben poder movilizar recursos cognitivos -y también técnicos- como el conocimiento de sus contextos específicos, y en particular poder utilizar equipamientos que les permitan realizar un trabajo homogéneo con el de los otros sitios de observación.

Luego, desde la coordinación del programa se procesan los resultados (en algunos proyectos ya hay un primer grado de procesamiento local), y se elaboran los productos, que pueden tener la forma de protocolos de intervención, recomendaciones de política (a organismos internacionales, a la Unión Europea o a gobiernos nacionales), y también bajo el formato de artículos científicos o eventualmente de transferencia a empresas privadas, si bien en el caso de los proyectos de medio ambiente su participación suele ser menor que en otros campos temáticos.

\section{d) América Latina resulta un contexto importante} -y a veces indispensable- para la observación/experimentación

Es el caso del desarrollo de organismos genéticamente modificados (OGM) que corresponden al consorcio KBBE-1. Si bien en Europa están autorizados los ensayos en laboratorio, es prácticamente imposible realizar ensayos de campo a una escala importante. En cambio, en América Latina existen muy pocas restricciones para ensayos de OGMs, siempre que se respeten algunas normas técnicas básicas. En este sentido, los consorcios que desarrollan actividades que están prohibidas o son muy difíciles en Europa deben conseguir, imperiosamente, un terreno fuera de su región donde poder desarrollar dichos ensayos. Algo similar ocurre en los consorcios KBBE-4 y Salud-1, en donde están implicadas pruebas clínicas con pacientes que, si bien 
no están prohibidas en la Unión Europea, tienen regulaciones mucho más rígidas que en Argentina y Brasil, donde se han desarrollado dichos ensayos en ambos consorcios, con menores controles y, por lo tanto, mayor celeridad en la obtención de resultados.

Tipo de actividad de los grupos latinoamericanos y beneficios obtenidos

En la muestra de los consorcios de investigación que hemos seleccionado para nuestra indagación, los tipos de actividades realizadas por los grupos latinoamericanos que prevalecen, en todos los campos temáticos, son la recolección y sistematización de datos, y el trabajo técnico, ya sea rutinario o innovador. Ello nos refiere a un tipo de inserción específica en la planificación de la investigación que es consistente con las motivaciones de los líderes europeos para el reclutamiento de dichos grupos: como vimos, en la mayor parte de los proyectos predominan las razones referidas a los sitios de observación de fenómenos, sean éstos específicos de América Latina (aunque con intereses globales), o las manifestaciones locales de fenómenos de un alcance mucho más amplio.

A ello se agregan dos datos muy importantes: por un lado, en la mayoría de los casos los grupos latinoamericanos fueron invitados a participar de los consorcios una vez que las grandes líneas del plan de trabajo ya estaban diseñadas y, por lo tanto, su participación en el diseño metodológico y en la distribución de tareas ha sido muy escasa. Hay sólo tres excepciones: los casos de Salud-1 y 4 y KBBE-6, donde los grupos latinoamericanos participaron activamente del diseño, según nos refirieron, en las entrevistas, los responsables europeos de cada proyecto.

Por otro lado, sólo en dos de los consorcios analizados los grupos latinoamericanos estuvieron a cargo de la coordinación de un working package, es decir, de la administración de una parte de los recursos asignados al proyecto. Se trata de Salud-4 y KBBE6 (que son también dos de los proyectos en donde los grupos participaron del diseño general de la investigación) y, en ambos casos, se trata de grupos de Brasil, muy prestigiosos, con una muy fuerte tradición en investigación y gran visibilidad, lo que los constituye en espacios de referencia internacional (FioCruz y EMBRAPA). ${ }^{6}$

En relación con la participación de las empresas, en todos los consorcios analizados (con la excepción de 3: ENV-1; ENV-4 y ENV-5) participan firmas de países europeos y en dos proyectos participan empresas latinoamericanas (una chilena y una argentina, respectivamente). Debemos señalar que, por disposiciones de la Unión Europea, las empresas que participan de los consorcios deben ser pequeñas y medianas. Según las declaraciones de los directores de los consorcios, ninguna de las empresas participantes en estos consorcios ha hecho aportes de fondos específicos para la investigación, sino que sus aportes son más bien "en especie", por ejemplo, a través de la provisión de compuestos químicos, capacidad de procesamiento de datos, actividades de marketing y difusión, o equipamientos específicos. Intentamos indagar en todos esos proyectos cómo son los acuerdos para la propiedad industrial y para la explotación de los resultados de las investigaciones, pero resultó imposible, porque son disposiciones reservadas.

Dicho esto, debemos destacar algunas particulari-

\footnotetext{
${ }^{6}$ La Fundación Oswaldo Cruz es una institución pública destinada a la investigación, la formación y la generación de políticas públicas en salud. Es la institución más importante, más antigua y más prestigiosa del país en el campo de la salud. La Corporación Brasileña de Investigación Agrícola (Embrapa), vinculada al Ministerio de Agricultura de Brasil, es una empresa de innovación tecnológica enfocada en generar conocimiento y tecnología para la agricultura brasileña. Es la mayor institución de investigación y aplicación de conocimiento agrícola en América latina.
} 
dades según los diversos campos temáticos-disciplinarios:

De los proyectos de salud, dos están orientados a la enfermedad de Chagas, una endemia propia de América Latina, y cuyos grupos más activos en el mundo corresponden a investigadores de Brasil, Argentina y, en menor medida, Colombia. En estos consorcios es donde la participación en el diseño de las investigaciones, y en las diferentes etapas es realmente significativo, y uno de ellos (Salud-4) es de los poquísimos casos en que el grupo latinoamericano administra un work package. De hecho, es también uno de los pocos que señala que el principal beneficio para el grupo es en términos económicos, ya que "Ios recursos que administran son muy importantes".

Asimismo, todos los proyectos de salud implican la participación de empresas, por lo general laboratorios farmacéuticos. Sin embargo, una sola de esas empresas es latinoamericana. Así, cuando intentamos indagar quién se haría cargo de la industrialización de los resultados, descubrimos que sería un laboratorio industrial europeo quien estaría a cargo de la producción de nuevos fármacos, mientras que el latinoamericano sólo se ocuparía de la distribución.

Por otro lado, en el área de salud, casi todos los grupos latinoamericanos tienen vínculos con pacientes y sólo en el caso de Salud-2, el grupo latinoamericano (chileno, en este caso) aporta la propiedad de compuestos de interés biológico y la capacidad de desarrollar análisis de big data.

No debemos pensar que en el campo de la salud las tareas de "recolección" y de "sistematización" de datos son actividades meramente técnicas, sin contenido científico, ya que, al tratarse de desarrollos experimentales, las capacidades científicas resultan fundamentales. Sin embargo, se trata de actividades subordinadas a una capacidad centralizada de procesamiento de la información, siempre localizada en alguno de los centros europeos.

En KBBE la situación es bastante similar: en general se refiere a procesos de investigación en agricultura y agroindustria, donde son determinantes la disponibilidad de recursos cognitivos sobre el medio local, y las fuertes tradiciones de investigación de los grupos latinoamericanos. Así, dos de los proyectos se orientan a hacer ensayos de campo en sus propios países, ya que en un caso ellos están prohibidos en Europa (KBBE-1) y en el otro las regulaciones son más laxas en nuestra región (KBBE-4). Otros grupos también se dedicaron a hacer ensayos clínicos (KBBE-5) o de campo (KBBE-6), actividades que son claramente predominantes.

Curiosamente, en KBBE, los grupos que desarrollan actividades de mayor complejidad científica son ambos de Chile: uno es un centro veterinario dedicado a la producción de vacunas (KBBE-3), y el otro es experto en una técnica de referencia internacional para la valoración de bio-residuos (KBBE-2).

En el campo de la investigación sobre medio ambiente, que tiene una menor tradición relativa en América Latina en relación con los dos campos anteriores (salud y agricultura), todos los grupos identificados realizan actividades de recolección de información y producción de datos, y en todos los casos según protocolos ya establecidos. Los datos generados por los grupos latinoamericanos se orientan a objetivos diferentes: sistematizar la concentración de mercurio en las aguas de diversas regiones (ENV1); utilizar modelos globales con puntos de observación específicos (ENV-2 y ENV-4); generar modelos conceptuales (ENV-3); o proponer políticas y herramientas de intervención (ENV-6). Sin embargo, la participación de los científicos de nuestra región en esos objetivos globales es muy baja o inexistente, ya que a menudo sus prácticas se detienen luego del procesamiento y la sistematización de los datos generados. 
En cuanto a los beneficios de la investigación para los grupos latinoamericanos, los hallazgos son concluyentes: el mayor beneficio se observa en términos del aumento de las publicaciones internacionales. A ello se le agrega la posibilidad de interactuar con grupos de investigación prestigiosos en el plano internacional. Contrariamente a lo que suponíamos, la obtención de fondos no aparece como el primer beneficio para la mayoría de los grupos latinoamericanos, según lo que declaran los coordinadores europeos. Ello puede deberse a diversas razones: primero, que al tratarse de grupos de élite, ya tengan cubiertas sus necesidades fundamentales de financiamiento; segundo, que haya beneficios intangibles, como el acceso a metodologías de punta, a compartir bases de datos internacionales, o a otros conocimientos tácitos; tercero, a que si bien la magnitud de recursos generales de los consorcios sea relativamente importante, la parte proporcional que reciben los latinoamericanos (con las pocas excepciones en que son, a su vez, administradores) no se perciba como significativa, en términos comparados con otros beneficios.

\section{Elementos para una tipología}

Para finalizar, y en función de todo lo anterior, queremos proponer una tipología preliminar de las diversas modalidades de inserción de los grupos latinoamericanos en consorcios internacionales, sustentada en el trabajo empírico. Para ello, definimos 4 tipos de participación, y los mostramos a partir del ejemplo concreto de uno de los consorcios analizados.

- Tipo 1: Participación en función de los recursos locales en América Latina indispensables para la investigación.

- Tipo 2: Participación por "subcontratación" para contribuciones específicas a una investigación global o para generar regulaciones.
- Tipo 3: Participación en función de la reputación internacional del grupo de América Latina, o conocimientos específicos necesarios para la investigación.

- Tipo 4: Participación en función de la posibilidad de hacer ensayos (clínicos, agrícolas) menos regulados en América Latina que en Europa.

Veamos brevemente cada una de estas modalidades:

- Tipo 1: Consorcio coordinado por un grupo catalán: pretende testear el perfil tóxico del Benznidazol, única droga existente para el tratamiento de Chagas.

El grupo brasileño (conformado por laboratorios de FioCruz y de la Universidad Federal de Ouro Preto) se dedica al diseño y puesta en marcha de ensayos clínicos. Según el coordinador, "son una pieza fundamental: el ensayo está basado exclusivamente en los datos producidos por los grupos brasileños (con enfermos de Chagas)" (entrevista con el coordinador del grupo, mayo de 2016). Se ocupan también de los modelos in vivo. El grupo argentino (que pertenece a la Administración Nacional de Laboratorios e Institutos de Salud, ANLIS) se ocupa de testear las nuevas moléculas alternativas al benznidazol, y su contribución es también "indispensable, gracias a su experiencia con T. cruzi".

A pesar de su importancia aparente, los grupos de América Latina no participaron en absoluto en la definición del proyecto (objetivos, métodos, o distribución de tareas).

- Tipo 2: Consorcio coordinado por un grupo inglés: se trata de analizar la polución de las aguas de los ríos por diferentes tipos de virus, como consecuencia del cambio climático.

El proyecto estudia diversos ríos europeos, y quiere contrastar con una región tropical (estudios en la cuenca del Amazonas). El grupo brasileño (Universidad Federal de Río de Janeiro) fue convocado 
porque es "muy respetado internacionalmente", pero sobre todo porque "era necesario (y oportuno) tener una localización tropical, y ellos tienen acceso al Amazonas". Su trabajo fue "tomar muestras según los protocolos, hacer las mediciones -que es un trabajo delicado- y generar los informes correspondientes" (entrevista con el coordinador del proyecto, abril de 2016).

El grupo coordinador ya había tenido dos pedidos de la Comisión Europea (y 2 proyectos europeos) para elaborar un policy report sobre la epidemiología de los ríos y las mediciones de concentración viral.

- Tipo 3: Consorcio coordinado por un grupo irlandés: encontrar un tratamiento gracias al micro RNA (pequeñas moléculas celulares) para casos en los cuales los medicamentos tradicionales no son eficaces para tratar la epilepsia.

El grupo brasileño (Universidad de Campinas) fue convocado porque es "muy conocido internacionalmente, con publicaciones importantes en el campo de la epilepsia. Sobre todo, son muy fuertes en los aspectos genéticos y lo que queríamos en este proyecto era mirar la genética de un tipo particular de gen en la epilepsia. Ese grupo brasileño tiene realmente competencias increíbles en el campo de la genética de la epilepsia" (entrevista con el coordinador del proyecto, mayo de 2016).

Por otro lado, para el consorcio era muy útil ensayar con "otras poblaciones -genéticamente hablandofuera de Europa (...) y es por eso que imaginamos hacer ensayos en USA y en Brasil" (el grupo está ligado a una clínica en Sao Paulo donde disponen de enfermos). Sin embargo, el trabajo genético fuera de Europa fue coordinado por un investigador estadounidense (Columbia University).

- Tipo 4: Consorcio coordinado por un grupo italiano: el objetivo es evaluar el impacto ambiental y económico posible del cultivo de OGMs.
Uno de los cultivos que se evalúa en este proyecto es la papa transgénica, tema en el cual el grupo argentino del Instituto Nacional de Tecnología Agropecuaria (INTA) trabajó durante varias décadas. La tarea del grupo argentino es en particular trabajar para la validación de la metodología de control en las áreas en las que los OGMs son cultivados a gran escala. Una primera validación de los métodos se prevé en el Reino Unido, pero es necesario probarlo en una producción de gran escala, lo que está permitido en América Latina pero no en Europa.

\section{Conclusiones}

A través del análisis empírico pudimos constatar que, mientras que las relaciones científicas se van complejizando, y la participación de grupos de investigación latinoamericanos en consorcios internacionales es cada vez mayor, la estructura básica de esas relaciones sigue organizándose en torno de modalidades de la "integración subordinada": las actividades que más emprenden los investigadores de América Latina en la división del trabajo en el interior de los consorcios de investigación es la producción, organización y sistematización de datos. Mientras tanto -con pocas excepciones- los grupos europeos tienden a concentrar el diseño de las investigaciones, tanto en términos teóricos como, sobre todo, metodológicos, así como la capacidad de centralizar los datos generados, y la de producir interpretaciones conceptuales.

En la medida en que casi todos los grupos de investigación latinoamericanos (con las excepciones mencionadas) son invitados a participar de los consorcios una vez que el diseño de la investigación ya ha sido establecido, la capacidad para orientar los resultados de esos trabajos hacia necesidades o un uso potencial en América latina resulta extremadamente improbable. 
Por otro lado, y tal como señalamos en el acápite anterior, en casi todos los consorcios participan empresas europeas. Aunque los acuerdos sobre propiedad industrial y explotación de resultados son reservados, es plausible deducir que dichos acuerdos implican que, de obtenerse resultados explotables comercialmente, ellos serán aprovechados en primer lugar por las empresas participantes de dichos consorcios y, enseguida, por otras empresas europeas. Así, la industrialización de los conocimientos si tiene lugar- habrá de desplegarse prioritariamente en el continente europeo.

Pudimos observar que el propósito declarado por la Unión Europea de "mejorar la calidad, el alcance y la masa crítica de la investigación" (European Commission, 2008) implicó la creciente participación de investigadores latinoamericanos, quienes aportan capacidades de investigación especializada en campos temáticos específicos, que se suman a los recursos humanos específicamente europeos. Ello ocurre, a diferencia del pasado, sin que se produzcan migraciones científicas permanentes o de larga duración, sino que los grupos latinoamericanos trabajan desde sus países, y aportan para ello, además, las infraestructuras ya existentes allí.

En este contexto, se genera un efecto de "proximidad cultural" y de comunidades ampliadas, entre pares de ambas regiones, que alimenta la cantidad de conocimientos disponibles para un uso potencial por parte de Europa.

La Unión Europea estableció el propósito de mejorar la competitividad, enfrentar desafíos sociales globales y respaldar a los países menos desarrollados en la construcción de capacidades en ciencia y tecnología. Digamos rápidamente que, dadas las características del importante desarrollo relativo de los grupos científicos de los países considerados, el fortalecimiento de las capacidades de esos países sin dudas ocurre como fruto de la cooperación ana- lizada, pero no es un elemento determinante (como lo sería en el caso de países con menor desarrollo). En cambio, es claro que la naturaleza de los proyectos considerados y sus dinámicas permiten fortalecer una visión global por parte de la Unión Europea, tanto a través de "puntos de observación" de diversos fenómenos globales en América Latina (como el cambio climático), como la posibilidad de hacer ensayos en terrenos con menos regulaciones, o aún por la posibilidad de generar conocimientos de cuestiones locales que completen una visión más amplia, o que contribuyan a generar regulaciones en Europa.

Finalmente, esperamos que la tipología propuesta -preliminar- constituya un punto de partida para profundizar el análisis sobre la participación de grupos latinoamericanos en consorcios internacionales y, más genéricamente, acerca de algunas modalidades sobre las que se estructuran las relaciones entre los centros hegemónicos de producción de conocimientos, y los contextos semi-periféricos. También es nuestra aspiración que la tipología pueda ser enriquecida con nuevas investigaciones empíricas, que tomen en cuenta, además de la cooperación con Europa, los vínculos financiados por las diversas agencias de los Estados Unidos, y también por modalidades de cooperación menos formalizadas, para observar si siguen un patrón similar a aquéllas que observamos aquí o si es posible identificar modalidades distintas.

\section{Bibliografía}

Adams, J. (2012). Collaborations: The rise of research networks. Nature, 490: 335-336.

Adams, J. (2013). Collaborations: The fourth age of research. Nature, 497: 557-560.

Boekholt, P; Edler, J; Cunningham, P. y Flanagan, K. (2009). Drivers of international collaboration in re- 
search. Amsterdam, Technopolis Group.

Cardoza, G. y Fornés, G., (2011). International co-operation of Ibero-American countries in business administration and economics research: Presence in high-impact journals. European Business Review, 23(1), pp. 7-22.

De Filippo, D, Morillo, F. y Fernández. M. T. (2008). Indicadores de colaboración científica del CSIC con Latinoamérica en bases de datos internacionales. Revista Española de Documentación Científica, Vol 31, No 1.

European Commission (2008). Directorate-General for Research. Opening to the world: international cooperation in science and technology: report of the ERA expert group. Luxembourgo: Office for Official Publications of the European Communities.

Gaillard, J.F., (1994). North- South Research Partnership: Is collaboration possible between Unequal Partners. Knowledge, Technology \& Policy, 7(2).

Gaillard, J. y Arvanitis, RT. (2013). Science and technology collaboration between Europe and Latin America: towards a more equal partnership? In. Gaillard, J. y Arvanitis, R. (eds) Research Collaborations between Europe and Latin America. Mapping and Understanding partnership. Paris: Editions des Archives Contemporaines.

Gaillard, J.F; Gaillard. A.M. y Arvanitis, R. (2010). Mapping and understanding EURO-LAC International Cooperation in Science and Technology. EULAKS Document. Seventh Framework Programme.

Gómez, I., Fernández, M.T. y Sebastián, J., (1999). Analysis of the structure of international scientific cooperation networks through bibliometric indicators. Scientometrics, 44(3), pp. 441-457.

Katz, J. S., y Martin, B. R. (1997). What is research collaboration? Research Policy, 26.

Kreimer, P. (2019). Science \& Society in Latin Ameri- ca. Peripheral Modernities. New York: Routledge.

Kreimer, P. (2006). ¿Dependientes o integrados? La ciencia latinoamericana y la división internacional del trabajo. Nómadas Nro. 24.

Kreimer, P. y Levin, L. (2013). Scientific Cooperation between the European Union and Latin American Countries: Framework Programmes 6 and 7. En: Gaillard y Arvanitis (eds.) Research Collaborations between Europe and Latin America. Mapping and Understanding partnership. París: Editions des Archives Contemporaines.

Leydesdorff, L. y Wagner, C. (2008). International collaboration in science and the formation of a core group. Journal of Informetrics, 2(4), pp.317-25.

Losego, P. y Arvanitis, R., (2008). La science dans les pays non hégémoniques. Revue d'Anthropologie des Connaissances, Vol. 2, № 3

Miguel, S. y Moya-Anegón, F. (2009). La ciencia argentina bajo la lupa de los indicadores cienciométricos: una mirada crítica de la realidad científica argentina. La Plata: Al Margen.

Persson O, Glänzel W, Danell R, (2004). Inflationary bibliometric values: The role of scientific collaboration and the need for relative indicators in evaluative studies. Scientometrics; 60(3): 421-432.

Velho, L. (2002). North-South Collaboration and Systems of Innovation. The International Journal of Technology Management and Sustainable Development, 1(3).

Vessuri, H. (1996). Scientific Cooperation among Unequal Partners: the Strait Jacket of the Human Resource Base, en J. Gaillard, Coopérations Scientifiques Internationales, París: Éditions de l' ORSTOM. 